\section{OPEN ACCESS}

Edited by:

Roberto Truzoli,

University of Milan, Italy

Reviewed by:

Annika Gunst

Åbo Akademi University, Finland

Chunbo $L i$,

Shanghai Jiao Tong University, China

Felicitas Engel,

Heidelberg University

Hospital, Germany

*Correspondence:

Heng Wu

hengwu@tongji.edu.cn

†These authors share last authorship

Specialty section:

This article was submitted to

Psychology for Clinical Settings,

a section of the journal

Frontiers in Psychology

Received: 30 April 2020

Accepted: 03 June 2021

Published: 01 July 2021

Citation:

Chen Y, Fink $P$, Wei J, Toussaint A-K, Zhang L, Zhang Y, Chen H, MaX, Li W, Ren J, Lu W, Leonhart R, Fritzsche $K$ and Wu H (2021)

Psychometric Evaluation of the

Whiteley Index-8 in Chinese

Outpatients in General Hospitals.

Front. Psychol. 12:557662.

doi: 10.3389/fpsyg.2021.557662

\title{
Psychometric Evaluation of the Whiteley Index-8 in Chinese Outpatients in General Hospitals
}

\begin{abstract}
Yixiao Chen ${ }^{1}$, Per Fink ${ }^{2}$, Jing Wei ${ }^{3}$, Anne-Kristin Toussaint ${ }^{4}$, Lan Zhang ${ }^{5}$, Yaoyin Zhang ${ }^{6}$, Hua Chen ${ }^{7}$, Xiquan $\mathrm{Ma}^{8}$, Wentian $\mathrm{Li}^{9}$, Jie Ren ${ }^{10}$, Wei Lu ${ }^{11}$, Rainer Leonhart ${ }^{12}$, Kurt Fritzsche ${ }^{13 t}$ and Heng $\mathrm{Wu}^{1 * t}$

${ }^{1}$ Department of Psychosomatic Medicine, Shanghai Tongji Hospital, Tongji University School of Medicine, Shanghai, China, ${ }^{2}$ The Research Clinic for Functional Disorders and Psychosomatics, Aarhus University Hospital, Aarhus University, Aarhus, Denmark, ${ }^{3}$ Department of Psychological Medicine, Peking Union Medical College Hospital, Chinese Academy of Medical Sciences \& Peking Union Medical College, Beijing, China, ${ }^{4}$ Department of Psychosomatic Medicine and Psychotherapy, University Medical Center Hamburg-Eppendorf, Hamburg, Germany, ${ }^{5}$ Mental Health Centre, West China Hospital, Sichuan University, Chengdu, China, ${ }^{6}$ Department of Psychosomatic Medicine, Sichuan Provincial People's Hospital, University of Electronic Science and Technology of China, Chengdu, China, ${ }^{7}$ Department of Psychological Medicine, Zhong Shan Hospital, Fudan University, Shanghai, China, ${ }^{8}$ Department of Psychosomatic Medicine, School of Medicine, Dongfang Hospital, Tongji University, Shanghai, China, ${ }^{9}$ Department of Clinical Psychology, Wuhan Mental Health Center, Wuhan, China, ${ }^{10}$ Department of Rehabilitation, General Hospital of Jincheng Anthracite Coal Mining Group Co. Ltd., Jincheng, China, ${ }^{11}$ Department of Psychosomatic Medicine, Beijing Hospital of Traditional Chinese Medicine, Capital University, Beijing, China, ${ }^{12}$ Institute of Psychology, University of Freiburg, Freiburg, Germany, ${ }^{13}$ Department of Psychosomatic Medicine and Psychotherapy, Faculty of Medicine, Medical Center - University of Freiburg, Freiburg, Germany
\end{abstract}

Background: Excessive and persistent health anxiety is a common and disabling but often unrecognized illness. Therefore, screening patients for health anxiety is recommended in primary care. The aim of the present study was to examine the psychometric properties of an updated version of the eight-item Whiteley Index (WI-8) among outpatients in general hospitals in China.

Methods: The presented data were derived from a multicenter cross-sectional study. The Chinese version of the WI-8 was administered to a total of 696 outpatients. Cronbach's alpha was used to evaluate the internal consistency of the scale. The validity of the scale was evaluated based on factor analysis and correlation analyses. To assess the discriminant ability, receiver operating characteristic $(R O C)$ analysis was conducted.

Results: Cronbach's alpha was 0.937, and it decreased (0.925) after deleting the new 8th item. Factor analysis extracted one factor accounting for $69.2 \%$ of the variance. Moderate correlations were found (0.414-0.662) between the WI-8 and General Anxiety Disorder (GAD-7), Patient Health Questionnaire-9 (PHQ-9), Patient Health Questionnaire15 (PHQ-15) and Somatic Symptom Disorder B-criteria (SSD-12). The ROC curve indicated excellent discriminatory ability to discriminate among patients with health anxiety ( $A \cup C=0.822)$.

Conclusions: The new WI-8 version is a reliable and valid tool to screen for health anxiety in general hospital patients. We recommend the WI-8 as a useful screening tool for health anxiety.

Keywords: Whiteley Index, health anxiety, reliability, validity, ROC 


\section{BACKGROUND}

Health anxiety is defined by cognitive-behavioral researchers as worry about health ranging from mild concern to excessive preoccupation (Ferguson, 2009; Longley et al., 2010). Excessive and persistent health anxiety is a common and disabling condition that can result in substantial suffering, difficult doctorpatient relationships and high health care costs (Robbins and Kirmayer, 1996; Barsky et al., 2001; Fink et al., 2010; Sunderland et al., 2013; Bobevski et al., 2016). Moreover, health anxiety can become chronic and incapacitating, with a majority $(>60 \%)$ of cases still showing symptoms after several years of followup (Sadock, 2017). Health anxiety often remains unrecognized (Gureje et al., 1997; Conradt et al., 2006); thus, early detection is crucial for reducing such serious impairment. Screening for health anxiety among primary care patients has been proposed (Fink et al., 1999).

Health anxiety has often been referred to as hypochondriasis (American Psychiatric Association., 2000). However, the DSM-IV definition of hypochondriasis has received criticism because the criteria were too narrow to be applied in clinical practice (Fink et al., 2004). Therefore, the DSM-V replaced hypochondriasis, among others, with illness anxiety disorder (IAD) and somatic symptom disorder (SSD) (American Psychiatric Association, 2013), and the classifications of IAD and SSD have proven to be more reliable in detecting health anxiety than hypochondriasis (Newby et al., 2017). The difference between the two disorders lies in the severity of somatic symptoms. SSD diagnosis requires distressing and disabling somatic symptoms in criterion A, and related thoughts, feelings, and behaviors in criterion $\mathrm{B}$. IAD is characterized by nonexistent or relatively minor somatic symptoms, excessive fears of illness, and high levels of health anxiety (Sadock, 2017). Bailer et al. (2016) found no difference in health anxiety severity, other hypochondriacial characteristics, illness behavior, somatic symptom attributions, and physical concerns between IAD patients and SSD patients, whereas Newby et al. (2017) proposed that SSD patients show more severe health anxiety. Nevertheless, these studies have suggested that health anxiety is an important feature of IAD and SSD.

The Whiteley Index (WI) is a "classic" scale of hypochondria, which was first developed by Pilowsky in the 1960s. Based on the principle diagnosis criteria of hypochondriasis in DSM IV, the scale consists of 14 items in 3 dimensions: disease phobia, somatic preoccupation and disease conviction (Pilowsky, 1967). Subsequent study has indicated a wide variation of factor models when generalized to different populations (Speckens et al., 1996). Hence, various of derivative of WI were developed, among which the most widely applied version is the 7-item WI (WI-7) developed by Fink et al. via latent structure analysis (Fink et al., 1999; Tu et al., 2016; Laferton et al., 2017). Due

Abbreviations: DSM-IV, Diagnostic and Statistical Manual of Mental Disorder (4th Edition); DSM-V, Diagnostic and Statistical Manual of Mental Disorder (5th Edition); GAD-7, General Anxiety Disorder; IAD, Illness Anxiety Disorder; PHQ-9, Patient Health Questionnaire-9; PHQ-15, Patient Health Questionnaire15; ROC, Receiver Operating Characteristic; SSD, Somatic Symptom Disorder; SSD-12, Somatic Symptom Disorder B-criteria; WI, Whiteley Index. to the highly overlapped diagnosis criteria of hypochondria and other somatoform disorders, a more specific manifestation was investigated to disentangle hypochondria from somatoform disorder. In an interview study involving 701 participants, it was found that patients with health anxiety generally had the symptom "rumination," which is not common in patients with somatoform disorder (Fink et al., 2004). Thus, the new item on obsessive rumination, "Recurring thoughts about having a disease that are difficult to get rid of," was added to the original WI-7 (Carstensen et al., 2020). The updated 8-item (WI-8) may be more helpful in screening for health anxiety.

The aim of the present study was to examine the psychometric properties of an updated WI-8 version to better identify health anxiety in outpatients in general hospitals in China.

\section{METHODS}

\section{Study Design and Setting}

Data were derived from a multicenter cross-sectional study. The study sites were three medical settings, departments of traditional Chinese medicine (TCM), neurology/cardiology/gastroenterology (Biomedicine), and psychosomatic medicine (PSY), from nine tertiary grade A hospitals in Beijing, Kunming, Shanghai and Chengdu. Participants who had signed an informed consent were asked to complete questionnaires on sociodemographic information, psychological data, and other clinical characteristics. Afterwards, IAD and SSD were diagnosed using the Structured Clinical Interview for DSM-V (Research Version) (SCDI-5), a semistructured tool for the diagnosis of mental disorders from the DSM-5. To ensure consistency, all interviewers were mental health professionals familiar with DSM-5 classification and passed interview techniques training. They were blinded to the patients questionnaire results. The study was approved by the ethics committees of Peking Union Medical College Hospital (PUMCH) and the University Medical Centre, Freiburg, Germany (Protocol Number: S-K276).

\section{Patient Recruitment}

From May 2016 to March 2017, participants were consecutively recruited from TCM, Biomedicine, and PSY settings on randomly assigned screening days. Participation in the study was voluntary. All the participants endorsed informed consent.

The inclusion criteria were (1) being at least 18 years of age, (2) having sufficient reading and writing skills, (3) attending the hospital visit to receive care oneself, and (4) signing an informed consent form. The exclusion criteria included the following points: (1) a hospital visit for someone else, (2) communication difficulties or language barriers, (3) cognitive impairment, organic brain disorder, dementia, (4) psychosis, or (5) acute suicidal tendency.

Among 1,269 eligible patients, 502 patients refused to participate, 68 patients were excluded on the basis of the exclusion criteria, and 3 additional patients had missing WI-8 score data; therefore, a total of 696 patients were included in this study (see Figure 1). 


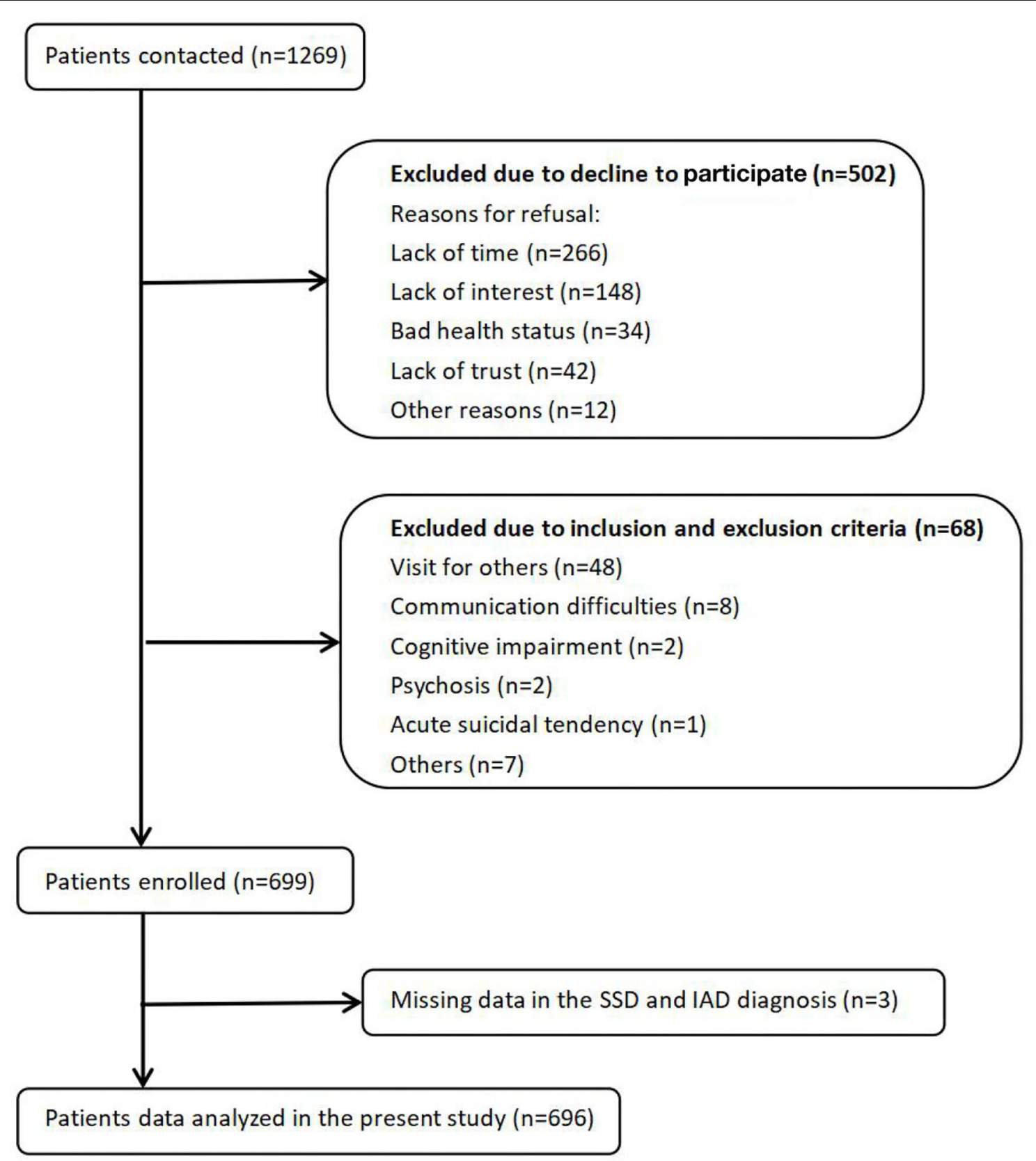

FIGURE 1 | Flow chart of patient enrollment.

\section{Assessment Instruments} Whiteley-8 (WI-8)

The WI- 8 is a self-reported scale that indicates the severity of health anxiety experienced over the previous 4 weeks. Each item is scored on a scale ranging from 1 to 5 ( $1=$ "no," $2=$ "a little," 3 = "some" 4 = "often," $5=$ "severe"). Fink et al. through a large number of interviews found that rumination could better distinguish health anxiety from other somatoform disorders, and therefore proposed this new WI version (Fink et al., 2004; Carstensen et al., 2020). Compared to the well-validated WI-7
(Lee et al., 2011; Tu et al., 2016; Laferton et al., 2017), the WI-8 extends an item, "Recurring thoughts about having a disease that are difficult to get rid of," to further detect obsessive rumination.

\section{Additional Questionnaires}

It is well-known that health anxiety commonly co-occurs with somatic symptoms, general anxiety, and depressive symptoms (Kroenke and Rosmalen, 2006). Moreover, these symptoms have distinguishing and additive effects on health-related quality of life, functional status, disability, and health care use (Spitzer 
et al., 1995; Kroenke et al., 2002; Löwe et al., 2008). Thus, we selected four additional questionnaires, the General Anxiety Disorder (GAD-7), Patient Health Questionnaire-9 (PHQ9), Patient Health Questionnaire-15 (PHQ-15), and Somatic Symptom Disorder B-criteria (SSD-12), to measure general anxiety, depression and physical symptoms.

(1) General Anxiety Disorder (GAD-7)

The GAD-7 is a brief, self-administered tool to screen for and estimate the severity of generalized anxiety disorders. The GAD7 contains 7 items, and each item is scored on a scale ranging from 0 to 3. Previous studies have reported that the scale has satisfactory reliability as well as factorial and concurrent validity (He et al., 2010).

(2) Patient Health Questionnaire-9 (PHQ-9)

The PHQ-9 is a 9-item self-report scale that is widely used to screen for depression. The scoring of each item ranges from 0 to 3 . The validity and reliability of this scale were confirmed in previous studies (Ran et al., 2017).

(3) Patient Health Questionnaire-15 (PHQ-15)

The PHQ-15 is a self-report questionnaire assessing somatic burden, including the 15 most typical somatic complaints in primary care. Each of the items is rated on a 3-point Likert scale ranging from 0 to 2 . This questionnaire has also shown good reliability and validity (Zhang et al., 2016).

(4) Somatic Symptom Disorder B-criteria (SSD-12)

The SSD-12 is a 12 -item self-report scale for the psychological criteria of SSD. Each of the items is rated on a 5-point Likert scale ranging from 0 to 5 . The reliability and validity of this instrument have been verified in another study (Hüsing et al., 2018; Li et al., 2020).

\section{Translation of the Questionnaires}

The WI- 8 was translated and back-translated from English into Chinese using a state-of-the-art procedure for test translation following the "ITC-Test Adaptation Guidelines" (version 2000) of the International Test Commission (ITC) (Merenda, 2006).

The Chinese versions of the PHQ-9, PHQ-15, GAD-7, and SSD-12 have been validated in Chinese samples and published in research papers (He et al., 2010; Zhang et al., 2016; Ran et al., 2017; Li et al., 2020). The questionnaire in Chinese language can be requested from the corresponding author.

\section{Statistical Analysis}

Data were analyzed using IBM SPSS Statistics 24 software and the web-based data science algorithm platform tool SPSSAU. Descriptive data that conformed to the normal distribution were expressed as mean \pm standard deviation, and assessed using the $t$-test; non-normally distributed data were expressed as median (quartile spacing), and assessed using the Mann-Whitney-U test. Qualitative variables were expressed as the frequency and percentage, and analyzed using Chi-square test. Given that IAD and SSD share the same characteristics in terms of health anxiety, 233 SSD patients, 12 IAD patients and 3 patients with both diagnoses were classified into the HA group $(n=248)$; the remaining patients constituted the non-HA group $(n=448)$.

The internal consistency of the WI-8 was assessed by Cronbach's alpha coefficient. The convergent and discriminant validity was evaluated based on Kendall's tau-b correlation analysis, since the scores of the scales do not satisfy the normal distribution. Exploratory factor analysis (EFA) was conducted to explore a factor structure of the WI-8. Confirmatory factor analysis (CFA) was performed to evaluate the effect of the new item- 8 on the construct validity of WI. The results of CFA were examined according to $\mathrm{Hu}$ and Bentler's suggestion that standardized root mean squared residual (SRMR) values $<0.08$, root mean squared error of approximation (RMSEA) values $<$ 0.06, and Comparative Fit Index (CFI) values higher than 0.95 are representative of a close fit of the model (Hu and Bentler, 1999). The diagnostic accuracy was evaluated by receiver operating characteristic (ROC) curve analysis with the area under the curve (AUC).

Statistical significance was assessed at 0.01 alpha level.

\section{RESULTS}

\section{Sample Characteristics}

The present study consisted of 696 patients (61.2\% female, $38.8 \%$ male, age $43 \pm 14.5$ years). The patients were predominantly urban dwellers (72.7\%), did not live alone (90.7\%), and were welleducated $(70.0 \%)$. However, the HA and non-HA groups did not differ significantly in terms of sociodemographic characteristics (see Table 1).

\section{Psychological Features}

The mean total score on the WI- 8 was $18.26 \pm 8.36$, with a median score of 16 , indicating a positive skewed distribution. The minimum value of the total score was 8 , suggesting that almost all the patients were experiencing health anxiety. Patients in the HA group also reported significantly higher GAD-7, PHQ9, PHQ-15, and SSD-12 scores compared to the non-HA group, suggesting that severe health anxiety is associated with general anxiety, depression, physical symptoms and SSD (see Table 1).

\section{Reliability}

Internal consistency was analyzed with Cronbach's alpha for the total scale score. Cronbach's alpha for the WI-8 was 0.937 , and it decreased (0.925) after deleting the 8th item. For the GAD-7, PHQ-9, PHQ-15, and SSD-12, the values were 0.936, $0.893,0.810$, and 0.954 , respectively, which also indicated acceptable reliability.

\section{Factor Analysis}

The construct validity of the questionnaire was evaluated by EFA and CFA. To determine the appropriateness of the factor analysis, the sample adequacy for extraction of the factors was confirmed through the Kaiser-Meyer-Olkin $(\mathrm{KMO})$ test $(\mathrm{KMO}=0.922)$ and Bartlett's test of sphericity $(p<0.001)$.

The EFA results revealed that one factor with an eigenvalue over 1 was extracted, accounting for $69.2 \%$ of the variance, and 
TABLE 1 | Sociodemographic information and clinical characteristics of the study sample $(n=696)$.

\begin{tabular}{|c|c|c|c|c|}
\hline & Total $(n=696)$ & Non-HA $(n=448)$ & HA $(n=248)$ & $P$ \\
\hline \multicolumn{5}{|l|}{ Sociodemographic information } \\
\hline Age & $43(23)$ & $43(22)$ & $42(23)$ & 0.918 \\
\hline Weight/kg & $60(15)$ & $60(15)$ & $59(17)$ & 0.085 \\
\hline Gender (man) & $270(38.8 \%)$ & $171(38.2 \%)$ & 99 (39.9\%) & 0.650 \\
\hline Gender (woman) & $426(61.2 \%)$ & $277(61.8 \%)$ & $149(60.1 \%)$ & \\
\hline Living situation (city) & $572(82.3 \%)$ & $374(83.5 \%)$ & $198(80.2 \%)$ & 0.272 \\
\hline Not living alone & $632(90.8 \%)$ & $406(90.6 \%)$ & $226(90.7 \%)$ & 0.826 \\
\hline Income higher than 8,000 yuan per month & $216(31.3 \%)$ & $145(32.6 \%)$ & $71(28.9 \%)$ & 0.312 \\
\hline Employed & $342(49.1 \%)$ & $233(52.0 \%)$ & $109(44.0 \%)$ & 0.042 \\
\hline High school or higher & $514(73.9 \%)$ & $340(75.9 \%)$ & $174(70.2 \%)$ & 0.099 \\
\hline $\mathrm{TCM}$ & $230(33.0 \%)$ & $182(40.6 \%)$ & 48 (19.4\%) & \\
\hline PSY & $242(34.8 \%)$ & $133(29.7 \%)$ & 109 (44.0\%) & \\
\hline Smoking currently & $100(14.4 \%)$ & 60 (13.4\%) & 40 (16.2\%) & 0.314 \\
\hline Alcohol everyday & $18(2.6 \%)$ & $12(2.7 \%)$ & $6(2.4 \%)$ & 0.843 \\
\hline Physical inactivity & 565 (81.2\%) & $361(80.6 \%)$ & 204 (82.3\%) & 0.588 \\
\hline Whiteley-8 & $16(11)$ & $14(8)$ & $23(13)$ & $<0.001$ \\
\hline GAD-7 & $5(8)$ & $4(6)$ & $9(9)$ & $<0.001$ \\
\hline PHQ-9 & $7(9)$ & $5(8)$ & $11(11)$ & $<0.001$ \\
\hline PHQ-15 & $9(7)$ & $7(6)$ & $12(8)$ & $<0.001$ \\
\hline SSD-12 & $11(20)$ & $6(13)$ & $24(16)$ & $<0.001$ \\
\hline
\end{tabular}

HA, health anxiety group (patients with illness anxiety disorder or somatic symptom disorder); Biomedicine, departments of neurology/cardiology/gastroenterology; TCM, department of traditional Chinese medicine; PSY, department of psychosomatic medicine; GAD-7, General Anxiety Disorder; PHQ-9, Patient Health Questionnaire-9; PHQ-15, Patient Health Questionnaire-15; SSD-12, Somatic Symptom Disorder B-criteria.

the factor loading ranged from 0.688 to 0.901 , indicating good construct validity of the 8 -item WI.

In the case of modification indices $(\mathrm{MI})>10$, the CFA of Fink's two-factor model of the original 6-item WI (Fink et al., 1999) showed that the model fit our data well (RMSEA $=0.056$, SRMR $=0.012, \mathrm{CFI}=0.997)$; While the one-factor 8-item model also showed satisfactory data fit (RMSEA $=0.057$, SRMR $=$ 0.019 , CFI $=0.993)$. In addition, the average variance extracted (AVE) was 0.681 , and the combined reliability (CR) was 0.943 (see Tables 2, 3).

\section{Discriminant Validity}

To examine the discriminant validity of the scale, Kendall's tau-b was used to study the relations among the WI-8, GAD-7, PHQ9, PHQ-15, and SSD-12 scores. The results in Table 4 show that the WI-8 score was moderately positively correlated with the GAD-7 (0.535), PHQ-9 (0.512), PHQ-15 (0.414), and SSD-12 (0.660) scores.

\section{Receiver Operating Characteristic Analyses}

ROC curve analysis was performed to assess the ability of the WI-8 to distinguish participants with health anxiety
TABLE 2 | Distributions and factor loadings of the WI-8.

\begin{tabular}{llccc}
\hline Item & Mean (SD) & Min & Max & Factor 1 \\
\hline 1 & $2.53(1.26)$ & 1 & 5 & 0.857 \\
2 & $2.36(1.30)$ & 1 & 5 & 0.885 \\
3 & $2.21(1.21)$ & 1 & 5 & 0.688 \\
4 & $2.28(1.33)$ & 1 & 5 & 0.901 \\
5 & $2.30(1.27)$ & 1 & 5 & 0.791 \\
6 & $1.76(1.07)$ & 1 & 5 & 0.761 \\
7 & $2.57(1.22)$ & 1 & 5 & 0.861 \\
8 & $2.24(1.36)$ & 1 & 5 & 0.887 \\
\hline
\end{tabular}

(IAD patients or SSD patients in our study). The WI8 showed excellent discriminatory ability, with an AUC of $0.822(p<0.001, \mathrm{CI}=0.789-0.854)$. The highest diagnostic accuracy for the WI- 8 was achieved by a cutoff value of 19 or higher. The sensitivity and specificity at an optimal cutoff point of $0 / 1$ were 0.730 and 0.777 , respectively (see Figure 2). 
TABLE 3 | Goodness-of-fit indices of the model for the Whiteley Index ( $n=348)$.

\begin{tabular}{|c|c|c|c|c|c|c|}
\hline Model & Factors & Items & $X^{2} / d f$ & RMSEA & SRMR & CFI \\
\hline$a^{\star}$ & 1 & 8 & 2.131 & 0.057 & 0.019 & 0.993 \\
\hline$b^{*}$ & 2 & 6 & 2.084 & 0.056 & 0.012 & 0.997 \\
\hline
\end{tabular}

$a^{*}$ Current study.

$b^{\star}$ Fink et al. (2004).

CFI, Comparative Fit Index; SRMR, standardized root mean squared residual; RMSEA, root mean squared error of approximation.

TABLE 4 | Correlation coefficients between the WI-8, GAD-7, PHQ-9, PHQ-15, and SSD-12.

\begin{tabular}{lllll}
\hline & WI-8 & GAD-7 & PHQ-9 & PHQ-15 \\
\hline WI-8 & & & & \\
GAD-7 & $0.535^{\star}$ & & & \\
PHQ-9 & $0.512^{\star}$ & $0.614^{\star}$ & & \\
PHQ-15 & $0.414^{\star}$ & $0.394^{\star}$ & $0.463^{\star}$ & \\
SSD-12 & $0.660^{*}$ & $0.492^{\star}$ & $0.502^{\star}$ & $0.392^{\star}$ \\
\hline
\end{tabular}

${ }^{*} P<0.001$

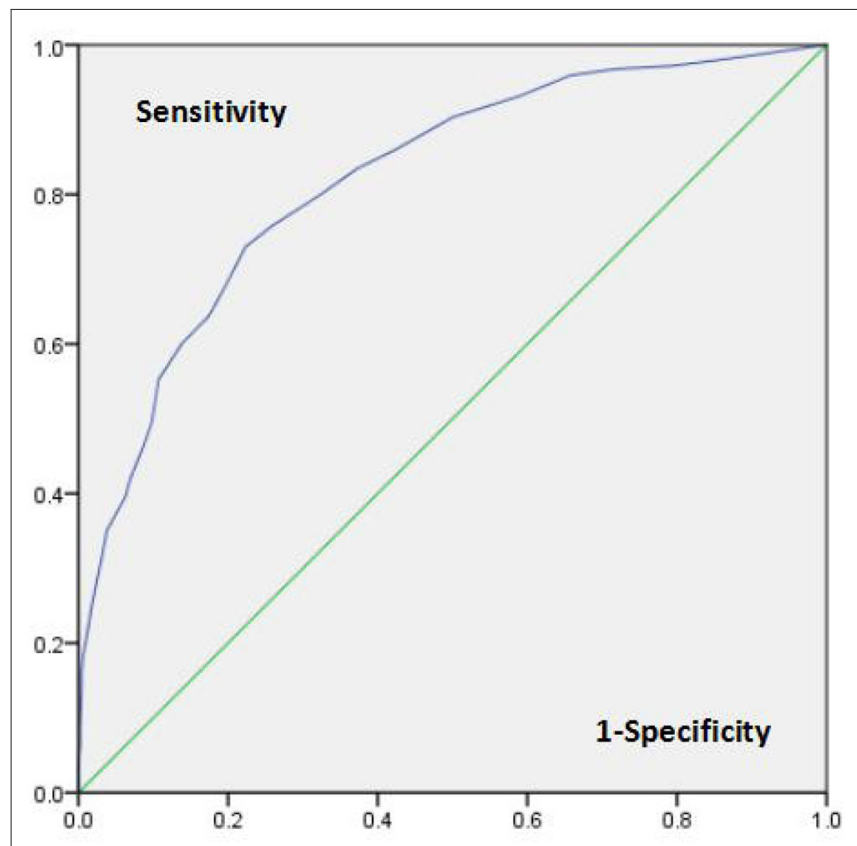

FIGURE 2 | ROC curve of the ability of the WI-8 to distinguish patients with IAD or SSD.

\section{DISCUSSION}

WI-8 was first developed by Fink et al. (2004) and Carstensen et al. (2020), and our study is the first to perform a psychometric evaluation of the Chinese version of the WI8 as a health anxiety screening scale. Participants were outpatients in general hospitals, predominantly city dwellers, well-educated and not living alone. The results revealed good reliability, validity and discriminant ability, suggesting that the WI- 8 could be used as an effective tool for screening health anxiety.

Cronbach's alpha of the WI-8 (0.937) was higher than that of all the previous versions (between 0.68 and 0.836) (Pilowsky, 1967; Welch et al., 2009; Lee et al., 2011; Hedman et al., 2015; Fergus et al., 2018). Moreover, Cronbach's alpha decreased after deleting the 8th item, indicating that the additional item 8 is not redundant, but increased the consistency of the scale. Various studies have reported the factor structure of WI and proposed unitary, 2-factor, and 3-factor models consisting of 6-14 items, indicating that the internal structure is unstable (Pilowsky, 1967; Welch et al., 2009). Therefore, the entire sample was randomly split into two parts: one group $(n=348)$ explores the WI- 8 factor structure by EFA, the other group $(n=348)$ compares the goodness of fit of different factor structures by CFA. EFA indicated a single factor model for WI-8, suggesting that the newly added item did not alter the one-factor structure of the original WI-7. Previous studies have also proposed that the original three factors of WI are highly related, and the one-factor model may be more suitable (Speckens et al., 1996). While Fink et al. (2004) pointed out that two isolated factors were more consistent with clinical characteristics, and they proposed a two-factor model including Illness Conviction subscale and Illness Worrying subscale. However, we compared the fitting index of the two models and found that the twofactor model was not significantly better than the singlefactor model. Furthermore, the AVE and CR indicated good convergent validity. It is possible that participants tended to rate all questions on the questionnaire consistently, resulting in a single structure.

The WI-8 had moderate correlations with the GAD-7, PHQ9, and PHQ-15, whereas in a study involving 200 patients and 240 healthy graduate students, the WI-7 had lower correlations with the GAD-7 (0.35), PHQ-9 (0.30), and PHQ-15 (0.33) (Güleç et al., 2012), perhaps because these psychological factors were more closely linked in patients than in healthy people. Furthermore, the WI- 8 had the weakest correlation with the PHQ-15 and the strongest correlation with the SSD-12. These findings are consistent with the IAD characteristics of light somatic symptoms and excessive health-related thoughts, and also suggest that health anxiety is an important feature of SSD. 
The WI-8 showed excellent discriminatory ability to distinguish patients with health anxiety, which is consistent with WI-6 (AUC $=0.83$ ) (Fergus et al., 2019), and superior to WI-7 (AUC $=0.660$ ) ( $\mathrm{Tu}$ et al., 2016). The results indicated that the WI- 8 offers a better way to identify persons with health anxiety, likely because of the additional item emphasizing recurring thoughts.

This study has several limitations. The stability of the WI8 over time could not be evaluated due to the cross-sectional study design. Additionally, the self-report scale could have been influenced by the educational background of the participants. Moreover, too few IAD patients could be analyzed as a subgroup, which affected the testing of the discriminant ability of the WI-8. Therefore, we grouped IAD patients and SSD patients into one group based on the similarity of their health anxiety characteristics. A lower proportion of IAD patients may be due to their mild physical symptoms and less hospital visits. Hence, future research with appropriate sample is planned to confirm and extend our findings in patients with general anxiety.

\section{CONCLUSION}

The latest version of the 8 -item WI has satisfactory reliability, validity and discriminant ability in general hospital outpatients in China. Therefore, we introduce the WI- 8 as an assessment tool for screening health anxiety.

\section{REFERENCES}

American Psychiatric Association (2013). Diagnostic and Statistical Manual of Mental Disorders, 5th Edn. Arlington, VA: American Psychiatric Association.

American Psychiatric Association. (2000). Diagnostic and Statistical Manual of Mental Disorders: DSM-IV-TR, 5th Edn. Washington, DC: American Psychiatric Association.

Bailer, J., Kerstner, T., Witthöft, M., Diener, C., Mier, D., and Rist, F. (2016). Health anxiety and hypochondriasis in the light of DSM-5. Anxiety Stress Coping 29, 219-239. doi: 10.1080/10615806.2015.1036243

Barsky, A. J., Ettner, S. L., Horsky, J., and Bates, D. W. (2001). Resource utilization of patients with hypochondriacal health anxiety and somatization. Med. Care 39, 705-715. doi: 10.1097/00005650-200107000-00007

Bobevski, I., Clarke, D. M., and Meadows, G. (2016). Health anxiety and its relationship to disability and service use: findings from a large epidemiological survey. Psychosom. Med. 78, 13-25. doi: 10.1097/PSY.0000000000000252

Carstensen, T. B. W., Ørnbøl, E., Fink, P., Pedersen, M. M., Jørgensen, T., Dantoft, T. M., et al. (2020). Detection of illness worry in the general population: a specific item on illness rumination improves the Whiteley Index. J. Psychosom. Res. 138:110245. doi: 10.1016/j.jpsychores.2020.110245

Conradt, M., Cavanagh, M., Franklin, J., and Rief, W. (2006). Dimensionality of the Whiteley Index: assessment of hypochondriasis in an Australian sample of primary care patients. J. Psychosom. Res. 60, 137-143. doi: 10.1016/j.jpsychores.2005.07.003

Fergus, T. A., Kelley, L. P., and Griggs, J. O. (2018). The Whiteley index6: an examination of measurement invariance among self-identifying Black, Latino, and White respondents in primary care. Assessment 25, 247-258. doi: $10.1177 / 1073191116645908$

Fergus, T. A., Kelley, L. P., and Griggs, J. O. (2019). Examining the Whiteley Index6 as a screener for DSM-5 presentations of severe health anxiety in primary care. J. Psychosom. Res. 127:109839. doi: 10.1016/j.jpsychores.2019.109839

Ferguson, E. (2009). A taxometric analysis of health anxiety. Psychol. Med. 39, 277-285. doi: 10.1017/S0033291708003322

\section{DATA AVAILABILITY STATEMENT}

The raw data supporting the conclusions of this article will be made available by the authors, without undue reservation.

\section{ETHICS STATEMENT}

The studies involving human participants were reviewed and approved by the ethics committees of Peking Union Medical College Hospital (PUMCH) and the University Medical Centre, Freiburg, Germany (Protocol Number: S-K276). The patients/participants provided their written informed consent to participate in this study.

\section{AUTHOR CONTRIBUTIONS}

HW, KF, PF, JW, LZ, and WLi contributed conception and design of the study. YZ, HC, XM, JR, and WLu contributed to data collection. YC and RL performed the statistical analysis. YC wrote the first draft of the manuscript. HW, KF, RL, and A-KT wrote sections of the manuscript. All authors contributed to manuscript revision, read, and approved the submitted vision.

\section{ACKNOWLEDGMENTS}

We thank all the participants.

Fink, P., Ewald, H., Jensen, J., Sørensen, L., Engberg, M., Holm, M., et al. (1999). Screening for somatization and hypochondriasis in primary care and neurological inpatients: a seven-item scale for hypochondriasis and somatization. J. Psychosom. Res. 46, 261-273. doi: 10.1016/S0022-3999(98)00092-0

Fink, P., Ørnbøl, E., and Christensen, K. S. (2010). The outcome of health anxiety in primary care. A two-year follow-up study on health care costs and self-rated health. PLoS ONE 5:e9873. doi: 10.1371/journal.pone.0009873

Fink, P., Ørnbøl, E., Toft, T., Sparle, K. C., Frostholm, L., and Olesen, F. (2004). A new, empirically established hypochondriasis diagnosis. Am. J. Psychiatry 161, 1680-1691. doi: 10.1176/appi.ajp.161.9.1680

Güleç, M. Y., Güleç, H., Simşek, G., Turhan, M., and Sünbül, E. A. (2012). Psychometric properties of the Turkish version of the patient health questionnaire-somatic, anxiety, and depressive symptoms. Compr. Psychiatry 53, 623-629. doi: 10.1016/j.comppsych.2011.08.002

Gureje, O., Ustün, T. B., and Simon, G. E. (1997). The syndrome of hypochondriasis: a cross-national study in primary care. Psychol. Med. 27, 1001-1010. doi: 10.1017/S0033291797005345

He, X. Y., Li, C. B., Qian, J., Cui, H. S., and Wu, W. Y. (2010). Reliability and validity of a generalized anxiety scale in general hospital outpatients. Shanghai Arch. Psychiatry 22, 200-203.

Hedman, E., Ljótsson, B., Andersson, E., Andersson, G., Lindefors, N., Rück, C., et al. (2015). Psychometric properties of Internet-administered measures of health anxiety: an investigation of the health anxiety inventory, the illness attitude scales, and the Whiteley Index. J. Anxiety Disord. 31, 32-37. doi: 10.1016/j.janxdis.2015.01.008

Hu, L. T., and Bentler, P. M. (1999). Cutoff criteria for fit indexes in covariance structure analysis: conventional criteria versus new alternatives. Struct. Equ. Model. 6, 1-55. doi: 10.1080/10705519909540118

Hüsing, P., Bassler, M., Löwe, B., Koch, S., and Toussaint, A. (2018). Validity and sensitivity to change of the somatic symptom disorder-B criteria scale (SSD-12) in a clinical population. Gen. Hosp. Psychiatry 55, 20-26. doi: 10.1016/j.genhosppsych.2018.08.006 
Kroenke, K., and Rosmalen, J. G. (2006). Symptoms, syndromes, and the value of psychiatric diagnostics in patients who have functional somatic disorders. Med. Clin. North Am. 90, 603-626. doi: 10.1016/j.mcna.2006.04.003

Kroenke, K., Spitzer, R. L., and Williams, J. B. (2002). The PHQ-15: validity of a new measure for evaluating the severity of somatic symptoms. Psychosom. Med. 64, 258-266. doi: 10.1097/00006842-200203000-00008

Laferton, J. A., Stenzel, N. M., Rief, W., Klaus, K., Brähler, E., and Mewes, R. (2017). Screening for DSM-5 somatic symptom disorder: diagnostic accuracy of selfreport measures within a population sample. Psychosom. Med. 79, 974-981. doi: 10.1097/PSY.0000000000000530

Lee, S., Ng, K. L., Ma, Y. L., Tsang, A., and Kwok, K. P. (2011). A general population study of the Chinese Whiteley-7 Index in Hong Kong. J. Psychosom. Res. 71, 387-391. doi: 10.1016/j.jpsychores.2011.05.013

Li, T., Wei, J., Fritzsche, K., Toussaint, A. C., Jiang, Y., Cao, J., et al. (2020). Validation of the chinese version of the somatic symptom disorder-B criteria scale for detecting DSM-5 somatic symptom disorders: a multicenter study. Psychosom. Med. 82, 337-344. doi: 10.1097/PSY.0000000000000786

Longley, S. L., Broman-Fulks, J. J., Calamari, J. E., Noyes, R., Wade, M., and Orlando, C. M. (2010). A taxometric study of hypochondriasis symptoms. Behavior. Ther. 41, 505-514. doi: 10.1016/j.beth.2010.02.002

Löwe, B., Spitzer, R. L., Williams, J. B., Mussell, M., Schellberg, D., and Kroenke, K. (2008). Depression, anxiety and somatization in primary care: syndrome overlap and functional impairment. Gen. Hosp. Psychiatry 30, 191-199. doi: 10.1016/j.genhosppsych.2008.01.001

Merenda, P. F. (2006). An overview of adapting educational and psychological assessment instruments: past and present. Psychol. Rep. 99, 307-314. doi: 10.2466/pr0.99.2.307-314

Newby, J. M., Hobbs, M. J., Mahoney, A. E., Wong, S. K., and Andrews, G. (2017). DSM-5 illness anxiety disorder and somatic symptom disorder: comorbidity, correlates, and overlap with DSM-IV hypochondriasis. J. Psychosom. Res. 101, 31-37. doi: 10.1016/j.jpsychores.2017.07.010

Pilowsky, I. (1967). Dimensions of hypochondriasis. Br. J. Psychiatry 113, 89-93. doi: 10.1192/bjp.113.494.89

Ran, C., Yu, W., Jianying, Y., and Lan, Z. (2017). Evaluation of the reliability and validity of phq-9 in general hospital inpatients. Sichuan Mental Health. 30:149-153.

Robbins, J. M., and Kirmayer, L. J. (1996). Transient and persistent hypochondriacal worry in primary care. Psychol. Med. 26, 575-589. doi: $10.1017 /$ S0033291700035650
Sadock, V. A. (2017). Kaplan and Sadock's Comprehensive Textbook of Psychiatry. Kaplan \& Sadocks Comprehensive Textbook of Psychiatry. Philadelphia, PA: Wolters Kluwer.

Speckens, A. E., Spinhoven, P., Sloekers, P. P., Bolk, J. H., and van Hemert, A. M. (1996). A validation study of the Whitely Index, the illness attitude scales, and the somatosensory amplification scale in general medical and general practice patients. J. Psychosom. Res. 40, 95-104. doi: 10.1016/0022-3999(95)00561-7

Spitzer, R. L., Kroenke, K., Linzer, M., Hahn, S. R., Williams, J. B., deGruy, F. V., et al. (1995). Health-related quality of life in primary care patients with mental disorders. Results from the PRIME-MD 1000 Study. JAMA 274, 1511-7. doi: 10.1001/jama.1995.03530190025030

Sunderland, M., Newby, J. M., and Andrews, G. (2013). Health anxiety in australia: prevalence, comorbidity, disability and service use. Br. J. Psychiatry 202, 56-61. doi: 10.1192/bjp.bp.111.103960

Tu, C. Y., Liao, S. C., Liu, C. Y., Chen, T. T., Chen, I. M., Lin, K. F., et al. (2016). Application of the Chinese version of the Whiteley index-7 for detecting DSM-5 somatic symptom and related disorders. Psychosomatics 57, 283-291. doi: 10.1016/j.psym.2015.12.010

Welch, P. G., Carleton, R. N., and Asmundson, G. J. (2009). Measuring health anxiety: moving past the dichotomous response option of the original Whiteley Index. J. Anxiety Disord. 23, 1002-1007. doi: 10.1016/j.janxdis.2009.05.006

Zhang, L., Fritzsche, K., Liu, Y., Wang, J., Huang, M., and Wang, Y., et al. (2016). Validation of the chinese version of the phq-15 in a tertiary hospital. BMC Psychiatry 16:89. doi: 10.1186/s12888-016-0798-5

Conflict of Interest: JR was employed by General Hospital of Jincheng Anthracite Coal Mining Group Co. Ltd., Jincheng, China.

The remaining authors declare that the research was conducted in the absence of any commercial or financial relationships that could be construed as a potential conflict of interest.

Copyright (C) 2021 Chen, Fink, Wei, Toussaint, Zhang, Zhang, Chen, Ma, Li, Ren, $\mathrm{Lu}$, Leonhart, Fritzsche and $W u$. This is an open-access article distributed under the terms of the Creative Commons Attribution License (CC BY). The use, distribution or reproduction in other forums is permitted, provided the original author(s) and the copyright owner(s) are credited and that the original publication in this journal is cited, in accordance with accepted academic practice. No use, distribution or reproduction is permitted which does not comply with these terms. 